\section{Endothelial cell loss and complication rates with combined Descemets stripping endothelial keratoplasty and cataract surgery in a UK centre}

SM Jones, MA Fajgenbaum and EJ Hollick

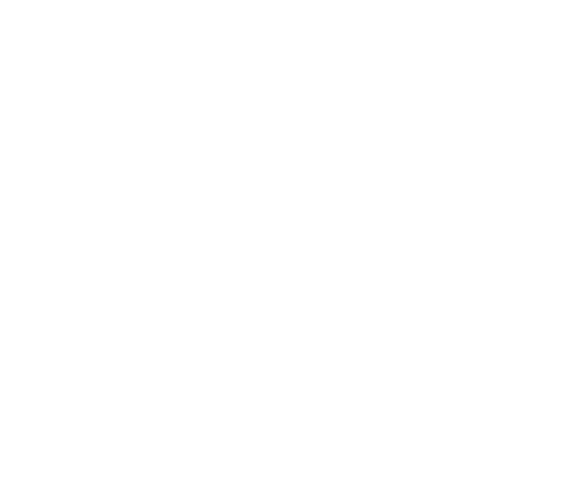

\begin{abstract}
Purpose To report 6-month, 1- and 2-year endothelial cell loss (ECL), intra- and postoperative complications in a large series of patients undergoing either Descemets stripping endothelial keratoplasty (DSEK) or a combined phacoemulsification and DSEK in a UK centre.

Patients and methods Patients undergoing DSEK with or without concurrent cataract surgery were included in this retrospective study. Surgeries were performed between January 2006 and May 2013. Main outcomes included intra- and postoperative complications and percentage ECL.

Results DSEK was performed in 226 eyes (210 patients). Of these, 141 eyes (126 patients) underwent DSEK alone and 85 eyes (84 patients) underwent DSEK combined with cataract surgery. Excluding complex anterior segment pathology the mean percentage ECL at 6,12 and 24 months was $40.5 \pm 13.4$, $45.1 \pm 14.6$ and $53.1 \pm 13.0$ in the DSEK group and $40.7 \pm 15.4,42.6 \pm 15.3$ and $49.6 \pm 16.5$ in patients undergoing the combined procedure, respectively. There was no significant difference in percentage ECL at 6 or 24 months between the two groups both in complex and routine cases. Intraoperative complications occurred in four patients undergoing DSEK and three undergoing combined procedure. Postoperative complication rates did not reach statistical significance between the groups.

Conclusion Mean ECL and complication rates were comparable at 6,12 and 24 months in routine cases undergoing concurrent DSEK with cataract surgery and those undergoing
\end{abstract}

DSEK. These data support the combined procedure in patients requiring both cataract surgery and endothelial keratoplasty. Further collaboration to report endothelial cell counts from other UK centres should be encouraged. Eye (2015) 29, 675-680; doi:10.1038/eye.2015.30; published online 13 March 2015

\section{Introduction}

There are differences of opinion as to whether cataract surgery should be performed concurrently or sequentially in patients requiring both cataract surgery and Descemets stripping endothelial keratoplasty (DSEK). ${ }^{1-3}$ The combined procedure avoids the risks involved with a second surgical procedure and may offer more rapid visual rehabilitation; however, surgeons may be concerned regarding the use of viscoelastic during the cataract surgery influencing graft attachment. ${ }^{1}$ Long-term outcomes, particularly endothelial cell counts, in the United Kingdom are infrequent in the literature. ${ }^{4}$ The purpose of this study is to report and compare mean percentage endothelial cell loss (ECL) and complication rates in a large series of patients undergoing DSEK with and without concurrent cataract surgery.

Patients undergoing DSEK with or without concurrent cataract surgery were included in this retrospective study. An institutional audit review board gave prior approval of the study, which was conducted in accordance with good clinical practices and the Declaration of Helsinki.
Materials and methods
Department of Ophthalmology, King's College Hospital NHS Foundation Trust, London, UK

Correspondence: EJ Hollick, Department of Ophthalmology, King's College Hospital NHS Foundation Trust, Denmark Hill, London SE5 9RS, UK Tel: + 44 (0)203 299 33797; Fax: +44 (0)203 2993738 E-mail: emma.hollick@ nhs.net

Received: 20 July 2014 Accepted in revised form: 26 January 2015 Published online: 13 March 2015

This work has been presented as a poster at the Royal College of Ophthalmologists Congress 2013. Abstract reference: ABS201211190636007742. 
All patients were operated on at a single institution under the supervision of a single surgeon (EJH). There were no exclusion criteria relating to preoperative or intraoperative factors. Both eyes of the same patient were permissible, as were re-grafts. Data were collated between January 2006 and May 2013 and entered prospectively at each follow-up visit, utilising an electronic web-based database 'eyedata.org'. Donor tissue was provided by the UK Transplant service and accepted with endothelial cell density $\left(\right.$ ECD) $>2000$ cells $/ \mathrm{mm}^{2}$.

Pupil dilation was performed in all patients preoperatively using $\mathrm{G}$ cyclopentolate $1 \%$ and $\mathrm{G}$ phenylephrine $2.5 \%$. Phacoemulsification and posterior chamber intraocular lens (IOL) implantation was performed through a temporal clear corneal incision followed by DSEK. The phacoemulsification technique included primary chop, insertion of a three-piece hydrophobic acrylic IOL into the capsular bag and extensive viscoelastic aspiration prior to DSEK. IOL selection took into account the expected hyperopic shift often induced by endothelial keratoplasty. ${ }^{2,5}$ The corneal epithelium was removed prior to biometry in those patients with significant preoperative corneal oedema, preventing accurate biometry measurement.

The procedure for DSEK surgery was standard and has been described in detail elsewhere. ${ }^{6}$ The DSEK technique included manual dissection of the donor material using an artificial anterior chamber and a Morlet lamellar dissector. An anterior chamber maintainer was utilised during donor insertion. Graft insertion was performed by one of three techniques: the Utrata forceps, the Busin glide (Moria USA, Doylestown, PA, USA) or the Tan Endoglide (Angiotech, Reading PA, USA/Network Medical Products, North Yorkshire, UK). The choice of insertion technique was dependent entirely on surgeon preference at the time of the procedure and not on patient-related factors.

Main outcomes measured included intra- and postoperative complications and percentage ECL at 6 months, 12 months and 2 years. The former included graft interface fluid, re-bubble, graft rejection and graft failure. The presence of any degree of interface fluid was included in the analysis for postoperative complications. A re-bubble procedure was performed in patients' with diffuse interface fluid, progression of interface fluid, interface fluid involving the central $6 \mathrm{~mm}$ of the graft or graft dislocation into the anterior chamber. All patients were managed using the same follow-up and drop regime. At each visit, a slit-lamp examination was performed and standard parameters (visual acuity, intraocular pressure, pachymetry) were recorded. Percentage ECL was obtained by measuring the donor central ECD using a corneal confocal microscope (Confoscan4; Nidek Technologies, Inc., Padova, Italy) at 6,
12 and 24 months and comparing this to the baseline preoperative Corneal Tissue Service (CTS) measurements.

Sub-group analyses were performed for complex versus routine grafts, based on preoperative and intraoperative characteristics. Complex grafts were defined as grafts with complicated anterior segments (Iridocorneal Endothelial syndrome, surgically managed glaucoma) and grafts with intraoperative complications.

Statistical analysis was performed using SPSS software version 20.0 (IBM Corp., Armonk, NY, USA). $\chi^{2}$-tests were utilised to compare categorical data for example complication rates, and independent sample $t$-test utilised for continuous data with significant differences reported at the $P<0.05$ level.

Multivariate analysis was performed to identify variables, which may influence the percentage ECL. Variables included patient age, gender, initial endothelial cell count (ECC), donor age, procedure performed (DSEK or phacoDSEK), previous glaucoma or glaucoma surgery, re-do procedures and complications peri/postoperatively (interface fluid, re-bubble, rejection, raised intraocular pressure).

\section{Results}

\section{Patient recruitment}

DSEK was performed in a total of 226 eyes (210 sequential patients). Of these eyes, 141 eyes (126 patients) underwent DSEK alone and 85 eyes (84 patients) underwent DSEK combined with cataract surgery. In the DSEK group, 49 eyes (39 patients) were complex grafts and 92 eyes (87 patients) were routine. In the DSEK combined with cataract surgery group, 9 eyes (9 patients) were complex grafts and 76 eyes (75 patients) were routine. Patient recruitment, demographics and operative details are provided in Figure 1 and Table 1, respectively. Of the 141 eyes undergoing DSEK alone, 134 were pseudophakic, 6 were aphakic and 1 patient remained phakic. In the latter patient, DSEK was performed in a blind eye (previous childhood trauma) for painful bullous keratopathy.

\section{Donor ECL}

Mean percentage ECL and mean exact cell counts at 6, 12 and 24 months for both groups for all cases and routine cases (in which complex anterior segment pathology was excluded) are shown in Table 2. Mean percentage ECL at 12 months was significantly lower in patients undergoing the combined procedure; however, this difference was not significant when adjusting for complex cases. There was no significant difference in percentage ECL at 6 or 24 months between patients undergoing DSEK and those 


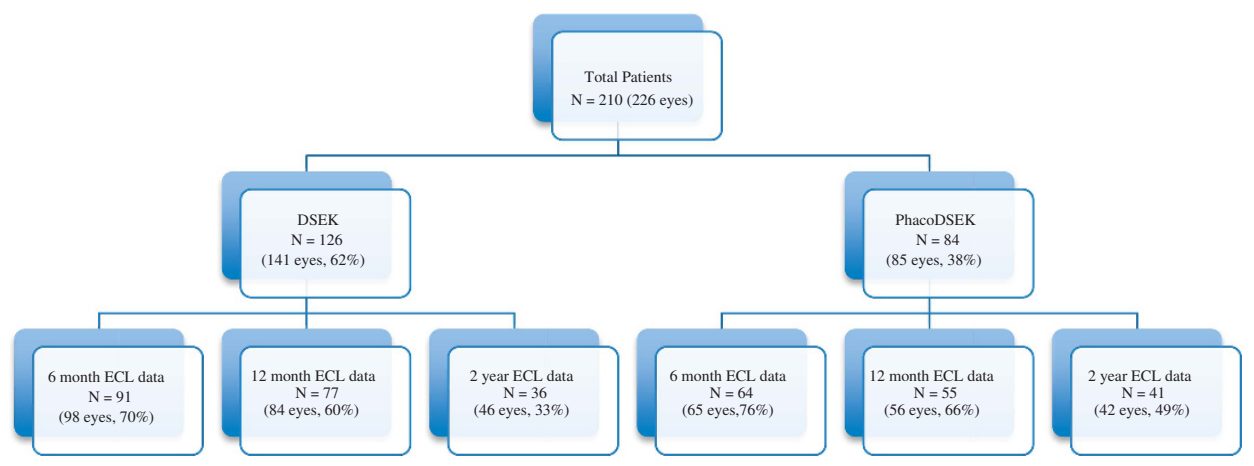

Figure 1 Patient recruitment. DSEK was performed in a total of 226 eyes (210 patients). Of these eyes, 141 eyes (126 patients) underwent DSEK alone and 85 eyes (84 patients) underwent DSEK combined with cataract surgery. Patient numbers (and number of eyes) with available ECL data at 6 months, 1 year and 2 years are shown in the above figure.

Table 1 Patient demographics

\begin{tabular}{|c|c|c|c|}
\hline & DSEK $\mathrm{N}=141$ eyes & PhacoDSEK $\mathrm{N}=85$ eyes & P-value \\
\hline Mean patient age (years $\pm S D$ ) & $73.63 \pm 12.29$ & $68.49 \pm 8.20$ & $P=0.001$ \\
\hline Mean donor age (years $\pm \mathrm{SD}$ ) & $70.35 \pm 20.87$ & $71.29 \pm 14.46$ & $P=0.713$ \\
\hline Gender, male & $61(43.3 \%)$ & $31(36.5 \%)$ & $P=0.314$ \\
\hline \multicolumn{4}{|l|}{ Primary indication } \\
\hline FED & $74(52.5 \%)$ & $79(92.9 \%)$ & \multirow[t]{7}{*}{$P \leq 0.001$} \\
\hline PBK & $39(27.7 \%)$ & 0 & \\
\hline ICE & $5(3.5 \%)$ & $2(2.4 \%)$ & \\
\hline Multiple surgery & $11(7.8 \%)$ & 0 & \\
\hline $\mathrm{ABK}$ & $6(4.3 \%)$ & 0 & \\
\hline Failed PK & $4(2.8 \%)$ & 0 & \\
\hline Other & $2(1.4 \%)$ & $4(4.7 \%)$ & \\
\hline Mean donor CTS preoperative ECC & $2562.94 \pm 276.62$ & $2546.19 \pm 167.76$ & $P=0.614$ \\
\hline Grade of surgeon consultant & $109(77.3 \%)$ cases & $68(80 \%)$ cases & $P=0.687$ \\
\hline \multicolumn{4}{|l|}{ Insertion technique } \\
\hline Tan endoglide & $80(56.7 \%)$ & $55(64.7 \%)$ & \multirow[t]{4}{*}{$P=0.662$} \\
\hline Busin glide & $39(27.7 \%)$ & $18(21.2 \%)$ & \\
\hline Forceps & $10(7.1 \%)$ & $6(7.1 \%)$ & \\
\hline Not recorded & $12(8.5 \%)$ & $6(7.1 \%)$ & \\
\hline
\end{tabular}

Abbreviations: ABK, Aphakic bullous keratopathy; CTS, Corneal Transplant Service; DSEK, Descemets stripping endothelial keratoplasty; ECC, endothelial cell count; FED, Fuchs Endothelial Dystrophy; ICE, Iridocorneal Endothelial; IOL, intraocular lens; PBK, Pseudophakic bullous keratopathy; PK, Penetrating keratoplasty.

undergoing the combined procedure both in complex and routine cases.

\section{Complications}

No complication occurred in any patient during phacoemulsification and lens implant. Intraoperative complications during the endothelial keratoplasty stage occurred in four patients undergoing DSEK and three patients undergoing the combined procedure. In the former, these included graft prolapse with reinsertion $(n=2)$, incarceration of the graft edge into the paracentesis requiring repositioning $(n=1)$ and dehiscence of the graft-host interface requiring resuturing in a patient who had previously undergone a full thickness penetrating keratoplasty $(n=1)$. In the latter group, intraoperative complications included graft prolapse with reinsertion $(n=1)$, extrusion of the AC maintainer during graft insertion $(n=1)$ and incorrect orientation of the graft requiring repositioning $(n=1)$.

Graft interface fluid, re-bubble and graft rejection occurred in 21 (14.9\%), 15 (10.6\%) and 19 (13.5\%) patients, respectively, undergoing DSEK and 11 (12.9\%), 11 (12.9\%) and $7(8.2 \%)$ patients undergoing combined procedure. These complication rates did not reach statistical significance between the two groups. Graft rejection was more frequent in the DSEK group; however, this did not reach statistical significance $(P=0.232)$ (Table 3 ). There were no primary graft failures in the entire cohort. 
Table 2 Mean percentage endothelial cell loss and mean endothelial cell count

\begin{tabular}{|c|c|c|c|c|c|}
\hline & \multicolumn{2}{|c|}{ DSEK $\mathrm{N}=141$ eyes } & \multicolumn{2}{|c|}{ PhacoDSEK $\mathrm{N}=85$ eyes } & \multirow[t]{2}{*}{ P-value } \\
\hline & $\% E C L$ & ECC cells $/ \mathrm{mm}^{2}$ & $\% E C L$ & ECC cells $/ \mathrm{mm}^{2}$ & \\
\hline \multicolumn{6}{|l|}{ All cases } \\
\hline 06 months & $44.51 \pm 14.85$ & $1438 \pm 412$ & $41.91 \pm 16.20$ & $1492 \pm 446$ & $P=0.292$ \\
\hline 12 months & $49.79 \pm 17.33$ & $1331 \pm 485$ & $43.71 \pm 16.21$ & $1451 \pm 447$ & $P=0.040$ \\
\hline 24 months & $54.46 \pm 14.41$ & $1201 \pm 389$ & $49.93 \pm 16.36$ & $1311 \pm 464$ & $P=0.171$ \\
\hline Routine cases & \multicolumn{2}{|c|}{ DSEK $\mathrm{N}=92$ eyes } & \multicolumn{2}{|c|}{ PhacoDSEK $\mathrm{N}=76$ eyes } & \\
\hline 06 months & $40.46 \pm 13.40$ & $1500 \pm 354$ & $40.72 \pm 15.39$ & $1503 \pm 458$ & $P=0.921$ \\
\hline 12 months & $45.13 \pm 14.59$ & $1425 \pm 451$ & $42.62 \pm 15.30$ & $1487 \pm 434$ & $P=0.385$ \\
\hline 24 months & $53.09 \pm 13.01$ & $1244 \pm 351$ & $49.63 \pm 16.45$ & $1320 \pm 473$ & $P=0.328$ \\
\hline
\end{tabular}

Abbreviations: \% ECL, percentage endothelial cell loss; ECC, endothelial cell count.

Table 3 Complication rates

\begin{tabular}{lccc}
\hline Complication & $\begin{array}{c}\text { DSEK } \\
\mathrm{N}=141\end{array}$ & $\begin{array}{c}\text { PhacoDSEK } \\
\mathrm{N}=85\end{array}$ & P-value \\
\hline Interface fluid & $21(14.89 \%)$ & $11(12.94 \%)$ & $P=0.683$ \\
Re-bubble procedure & $15(10.64 \%)$ & $11(12.94 \%)$ & $P=0.599$ \\
Graft rejection episode & $19(13.48 \%)$ & $7(8.24 \%)$ & $P=0.232$ \\
\hline
\end{tabular}

\section{Multivariate analysis}

Variables significantly associated with increased percentage ECL at 6, 12 and 24 months are shown in Table $4(\mathrm{a}-\mathrm{c})$. The effect of DSEK vs combined PhacoDSEK on percentage ECL was insignificant when adjusting for other variables at all three time points $(P=0.608,0.786$ and 0.251 at 6,12 and 24 months).

\section{Discussion}

Our findings of comparable ECL and complication rates when performing DSEK with and without concurrent cataract surgery support recent literature advocating combined DSEK with Cataract surgery. ${ }^{1,2}$ Our intraoperative complication rate $(7 / 226$ eyes, $3.1 \%)$ is comparable to recent literature. ${ }^{7}$ Mean ECL at 12 months was significantly lower in the combined procedure group when including all cases; however, this difference was not significant when adjusting for complex cases. Price et al reported that ECL was significantly less when DSEK was performed as a combined procedure. However, unlike our cohort, the graft insertion incision differed between the two groups. ${ }^{3}$

When comparing patient demographics, a significant difference between the two groups was found for patient age (significantly lower in the combined procedure group) and primary indication. The former could in part be explained by the number of patients with PBK in the
DSEK group being of an increased age having already undergone cataract surgery with subsequent decompensation. Primary indication for endothelial keratoplasty significantly differed between the two cohorts with the majority of cases performed for FED in the combined procedures group. There was no significant difference between the two cohorts for other patient demographics including insertion technique $(P=0.662)$.

Grafts of suitable thickness were achieved with manual dissection of the donor material utilising an artificial anterior chamber and a Morlet lamellar dissector. Published literature reports comparable ECL in donor tissue cut either manually or with a microkeratome. ${ }^{3,4,8}$

The postoperative ECC was obtained using confocal microscopy (Confoscan4). Both confocal and specular microscopy are validated objective methods to evaluate corneal endothelial morphology. As specular microscopy measures a smaller area of endothelial cells, one could perceive that this technique may over-count, particularly at lower cell counts. However, Farhan et al recently found no statistically significant difference between the SP-2000P specular microscopy and Confoscan 4 confocal microscopy, reporting both methods can be used interchangeably to measure ECD. ${ }^{9}$ The mean ECL was obtained by comparing the postoperative Confocal ECC with the preoperative ECC data measured by the CTS. The preoperative ECC were taken before these culturemedium preserved corneas were put into Dextran to deswell, which has been shown to lead to a loss of endothelial cells in excess of $8 \% .{ }^{10}$ Consequently, the actual preoperative ECC is likely to be lower than that provided by the eye bank. Despite this, our mean ECL are comparable with recent literature. ${ }^{2,5}$ Long-term outcomes, particularly ECCs in the UK, are infrequent in the literature. ${ }^{4,11}$

Following multivariate analysis, endothelial keratoplasty performed for indications other than Fuchs Endothelial dystrophy was associated with a higher 
Table 4 Multivariate analysis to determine factors influencing the percentage ECL at (a) 6 months, (b) 12 months and (c) 24 months

\begin{tabular}{|c|c|c|c|c|c|}
\hline \multirow[t]{2}{*}{ Model } & \multicolumn{2}{|c|}{ Unstandardised coefficients } & \multirow{2}{*}{$\frac{\text { Standardised coefficients }}{\text { Beta }}$} & \multirow[t]{2}{*}{$t$} & \multirow[t]{2}{*}{ Sig. } \\
\hline & $B$ & Std. error & & & \\
\hline \multicolumn{6}{|l|}{ (a) } \\
\hline Interface fluid & 16.771 & 3.560 & 0.334 & 4.711 & 0.000 \\
\hline Indication & 9.085 & 2.389 & 0.270 & 3.803 & 0.000 \\
\hline Initial ECC & -0.017 & 0.006 & -0.192 & -2.708 & 0.008 \\
\hline \multicolumn{6}{|l|}{ (b) } \\
\hline Rejection & 13.738 & 3.708 & 0.289 & 3.705 & 0.000 \\
\hline Indication & 11.051 & 3.006 & 0.287 & 3.676 & 0.000 \\
\hline Interface fluid & 8.171 & 3.836 & 0.161 & 2.130 & 0.035 \\
\hline \multicolumn{6}{|l|}{ (c) } \\
\hline Indication & 9.928 & 3.798 & 0.261 & 2.614 & 0.011 \\
\hline Re-bubble & 11.156 & 4.828 & 0.230 & 2.311 & 0.023 \\
\hline Initial ECC & -0.017 & 0.008 & -0.200 & -2.007 & 0.048 \\
\hline
\end{tabular}

percentage ECL at all times points. Other variables associated with higher percentage ECL included initial ECC at 6 and 24 months, graft dislocation at 6 and 12 months, graft rejection at 12 months and re-bubble at 24 months. Whether DSEK was performed with or without concurrent cataract surgery did not significantly increase percentage ECL when adjusting for other variables at each time point.

Our findings support combined endothelial keratoplasty with cataract surgery in appropriate patients. Our current practice is to offer the combined procedure to patients with cataract and evidence of corneal epithelial or stromal oedema. In those with FED without epithelial or stromal oedema, we recommend simple cataract extraction with lens implantation using a soft shell technique having explained the risk of postoperative decompensation and deliberately choosing a low myopia postoperative refractive target. Given the accommodative advantage of a crystalline lens in the younger population, patients younger than 50 years of age with visually insignificant cataract and a relatively deep anterior chamber should be offered DSEK alone. ${ }^{12-14}$ With endothelial keratoplasty continually evolving, it will be pertinent to establish the role of combined cataract surgery in these newer techniques. ${ }^{14,15}$

In conclusion, mean ECL and complication rates were comparable at 6, 12 and 24 months in routine cases undergoing concurrent DSEK with cataract surgery and those undergoing DSEK. To our knowledge, this is the largest UK data series reporting ECCs. It supports the combined procedure in patients requiring both cataract surgery and endothelial keratoplasty and enables comparison with existing published data from the global ophthalmic community. Further collaboration with other corneal units to report ECCs from UK centres should be encouraged.

\section{Summary}

What was known before

- There are differences of opinion as to whether cataract surgery should be performed concurrently or sequentially in patients requiring both cataract surgery and Descemets stripping endothelial keratoplasty.

- Long-term outcomes, particularly endothelial cell counts in the United Kingdom, are infrequent in the literature.

What this study adds

- To the best of our knowledge, this is the largest prospective data series reporting endothelial cell counts in the United Kingdom.

- Our findings support combined endothelial keratoplasty with cataract surgery in appropriate patients.

\section{Conflict of interest}

The authors declare no conflict of interest.

\section{Acknowledgements}

Dr Yanzhong Wang, Lecturer in Medical Statistics, King's College London.

\section{References}

1 Terry MA, Shamie N, Chen ES, Phillips PM, Shah AK, Hoar KL et al. Endothelial keratoplasty for Fuchs' dystrophy with cataract. Ophthalmology 2009; 116(4): 631-639.

2 Covert DJ, Koenig SB. New triple procedure: Descemet's stripping and automated endothelial keratoplasty combined with phacoemulsification and intraocular lens implantation. Ophthalmology 2007; 114(7): 1272-1277.

3 Price MO, Price FW. Endothelial cell loss after descemet stripping with endothelial keratoplasty; influencing factors and 2-year trend. Ophthalmology 2008; 115(5): 857-864. 
4 Rice A, Spokes DM, Anand S, Ball JL. Endothelial cell survival and graft profile analysis in Descemet stripping endothelial keratoplasty. Cornea 2011; 30(8): 865-871.

5 Lee WB, Jacobs DS, Musch DC, Kaufman SC, Reinhart WJ, Shtein RM. Descemet's stripping endothelial keratoplasty: safety and outcomes. a report by the American Academy of Ophthalmology. Ophthalmology 2009; 116(9): 1818-1830.

6 Gangwani V, Obi A, Hollick EJ. A prospective study comparing Endoglide and Busin Glide in Descemet's stripping endothelial keratoplasty. Am J Ophthalmol 2012; 153(1): 38-43.

7 Basak SK, Basak S. Complications and management in Descemet's stripping endothelial keratoplasty: analysis of consecutive 430 cases. Indian J Ophthalmol. 2014; 62(2): 209-218.

8 Terry MA, Chen ES, Shamie N, Hoar KL, Friend DJ. Endothelial cell loss after Descemet's stripping endothelial keratoplasty in a large prospective series. Ophthalmology 2008; 115(3): 488-496.

9 Al Farhan HM, Al Otaibi WM, Al Razqan HM, Al Harqan AA. Assessment of central corneal thickness and corneal endothelial morphology using ultrasound pachymetry, non-contact specular microscopy, and Confoscan 4 confocal microscopy. BMC Opthalmol 2013; 13(73): 1-8.

10 Borderie VM, Baudrimont M, Lopez M, Carvajal S, Laroche L. Evaluation of the deswelling period in dextran-containing medium after corneal organ culture. Cornea 1997; 16(2): 215-223.

11 Mearza AA, Qureshi MA, Rostron CK. Experience and 12-month results of descemet-stripping endothelial keratoplasty (DSEK) with a small-incision technique. Cornea 2007; 26(3): 279-283.

12 Huang T, Luo Y, Wang Y, Hu A, Gao N. Descemet's stripping with endothelial keratoplasty for special Fuchs endothelial dystrophy in phakic eyes. Ophthalmic Res 2011; 46(1): 44-49.

13 Price MO, Price DA, Fairchild KM, Price FW Jr. Rate and risk factors for cataract formation and extraction after Descemets stripping endothelial keratoplasty. Br J Ophthalmol 2010; 94: 1468-1471.

14 Anshu A, Price MO, Tan DTH, Price FW Jr. Endothelial keratoplasty: a revolution in evolution. Surv Opthalmol 2012; 57(3): 236-252.

15 Patel SV. Graft survival and endothelail outcomes in the new era of endothelial keratoplasty. Exp Eye Res 2012; 95(1): 40-47. 